\title{
Über die Trennung des Cers von Lanthan und Didym und seine quantitative Bestimmung in Gemischen mit letzteren. \\ Von \\ P. Mengeu.
}

\section{Die verschiedenen Trennungsverfahren.}

Mit Ausnahme der DeBray'schen partiellen Nitratzersetzung beruhen alle bisherigen Methoden der Abscheidung des Cers von den andern Erden der Cergruppe auf der Überführung desselben in das höhere Oxyd $\mathrm{CeO}_{2}$ und dessen Abscheidung entweder als schwerlösliches basisches Salz oder als Doppelsalz. Ausgehend vom Naturprodukt gelangt man, nach Abscheidung der anderen Elemente und nötigenfalls der Gadoliniterden, gewöhnlich zu den Oxalaten der Erden der Cergruppe, die durch starkes Glühen in die Oxyde übergeführt werden. Nach Ansicht der älteren Chemiker enthält nun das Oxydgemisch aufser den Sesquioxyden des Lanthans und Didyms (Neodyms und Praseodyms) das Cer sowohl als Dioxyd als auch als Sesquioxyd, was auch Schottlaender, ${ }^{1}$ der die Auer von Welsbach'sche Trennungsmethode einem näheren Studium unterworfen hat, noch annimmt. Dafs das reine Ceroxalat beim Giühen vollständig in reines Cerdioxyd übergeht, ist eine bekannte Thatsache, dafs dieses aber auch beim Glühen eines Gemisches der Oxalate stattfindet, wurde durch zu diesem Zweck angestellte Versuche erst unzweifelhaft nachgewiesen. Gemische von cerfreien Lanthan- und Didymsalzlösungen mit reinem Cersalz, deren Gehalte vorher bestimmt waren, wurden mit Oxalsäure gefällt und das Oxalat stark geglüht. Das Gewicht des resultierenden Oxydgemisches entsprach genau der Summe der Gewichte der Lanthan- und Didymsesquioxyde plus dem des Cerdioxyds, welches beim Glühen aus dem Sesquioxyd

${ }^{1}$ Ber. deutsch. chem. Ges. [1] 25, 378. 
des angewandten Salzes entstanden war. Die Menge des Dioxyds wurde auch direkt nach der weiter beschriebenen Methode bestimmt und aus ebenfalls später auseinanderzusetzenden Gründen etwas zu grols gefunden.

$0.2152 \mathrm{~g}$ eines Gemisches von $\mathrm{I}_{\mathrm{a}_{2}} \mathrm{O}_{3}$ und $\mathrm{Di}_{2} \mathrm{O}_{3}$ wurden in Form einer Sulfatlösung mit $0.1560 \mathrm{~g} \mathrm{Ce}_{2} \mathrm{O}_{3}$ (ebenfalls als Sulfat), entsprechend $0.1635 \mathrm{~g}$ $\mathrm{CeO}_{2}$, gemischt, mit Oxalsäure gefïllt, geglüht und $0.3785 \mathrm{~g}$ Oxyd statt $0.3787 \mathrm{~g}$ $(=0.2152+0.1635)$ gefunden. Die Analyse ergab darin $0.1762 \mathrm{~g} \mathrm{CeO}_{z}$ statt $0.1635 \mathrm{~g}$.

Dieses Verhalten des Ceroxalats, auch in Gemischen nit den andern Erden beim Glühen vollständig in das Dioxyd überzugehen, ist bei der leichten Oxydierbarkeit des Sesquioxyds vollkommen begreiflich. Wie W. MuthmanN ${ }^{2}$ vor kurzem nachgewiesen, ist es überhaupt unmöglich, aus reinem Cerokarbonat oder Oxalat durch Glühen - sogar im Wasserstoffstrome - das Sesquioxyd zu erbalten; regelmälsig resultierte Cerdioxyd, welches durch Wasserstoff nicht reduziert wird.

Zur Bestimmung des Gehaltes der angewandten Cerlösungen an Oxyd wurde sowohl mit Oxalsäure als auch mit Ammoniak und mit Natriumsuperoxyd gefällt, wobei die Resultate mit Ammoniak stets um ca. $1.5 \%$ geringer ausfielen als mit Oxalsäure und Natriumsuperoxyd, welch letztere sehr gut übereinstimmten. Dieses kann nur dadurch erklärt werden, dal's die Oxydhydrate dieser Erden in Ammoniak etwas löslich sind, oder es kann daran liegen, dafs der Ammoniakniederschlag beim Trocknen die Gestalt von kompakten Körnchen annimmt, welche beim Glühen sich nicht im Innern vollständig oxydieren können; letzteres ist jedoch nach dem oben Gesagten unwahrscheinlicher.

Das bisher Gesagte ist wichtig für die verschiedenen Abscheidungsverfahren des Cers. Nach den meisten. Methoden werden die durch Glühen der Oxalate erhaltenen Oxyde zunächst in starker Salpetersäure gelöst. Bis auf SCHOTTLIENDER war es allen frïheren Chemikern entgangen, dufs hierbei eine starke Sauerstoffentwickelung stattfindet, wobei das Cerdioxyd teilweise zu Sesquioxyd reduziert wird. Bei der in grölserer Menge freiwerdenden Kohlensäure, welche die geglühten Oxyde aus der Luft aufgenommen hatten, war der Sauerstoff übersehen worden. Hierin liegt auch der Grund der oben erwähnten irrigen Annahme, dals das Ceroxalat beim Glühen nicht

${ }^{1}$ Ber. deutsch. chem. Ges. (1898) 31, 1829. 
vollständig in das Dioxyd ühergehe; eine nachfolgende Oxydatior der salpetersauren Lösung mit verschiedenen Oxydationsmitteln is1 bei den verschiedenen Methoden erforderlich, nicht weil das $\mathrm{Cer}$ in dem Oxydgemisch zum Teil als Sesquioxyd vorhanden war, sondern weil das Dioxyd beim Lösen in starker Sal. petersäure teilweise reduziert wird. Die Sauerstoffentwickelung durch Salpetersäure, sowie die Chlorentwickelung durch Salzsäure beweisen, dafs das Cerdioxyd zur Gruppe der Polyoxyde vom Typus des $\mathrm{PbO}_{2}, \mathrm{MnO}_{2}$ u. s. w. gehört, jedoch als solches leichter Salze zu bilden im stande ist als letztere. Ebenso wie jene kann es aus der niedrigeren Oxydationsstufe durch verschiedene Oxydationsmittel, wie $\mathrm{Cl}, \mathrm{PbO}_{2}, \mathrm{KMnO}_{4}, \mathrm{Na}_{2} \mathrm{O}_{2}, \mathrm{H}_{2} \mathrm{O}_{2}$ u. dergl. dargestellt werden. Einen andern Charakter zeigt dagegen das später zu erwähnende Praseodymsuperoxyd, welches sich nicht mit Hilfe von Chlor aus dem Sesquioxyd darstellen lälst, und, wie PICcons ${ }^{1}$ richtig hervorhebt, zur Gruppe der eigentlichen Superoxyde vom Typus des $\mathrm{H}_{2} \mathrm{O}_{2}, \mathrm{BaO}_{2}$ u. s. w. gehört.

Um aus dem von Merck erhaltenen, sehr starke Didymabsorptionsstreifen zeigenden „cerium nitric." reines Ceroxyd darzustellen, wurde zunächst das von BüHRIG ${ }^{2}$ empfohlene kombinierte HolzManx-GiBbs'sche Verfahren angewandt, welches in der Abscheidung des Cers als basisches Sulfat und Oxydation des letzteren mit $\mathrm{Pb}_{3} \mathrm{O}_{4}$ besteht. Als Übelstände dieses Verfahrens erwiesen sich: 1. die grofse Löslichkeit des Sulfats in dem Waschwasser und die schwere Auswaschbarkeit des dichten Niederschlages - ein Nachtheil der Holzmann'schen Methode, 2. die grofse Löslichkeit des entstehenden Bleisulfats in der starken Salpetersäure - ein Nachteil des GibBs'schen Verfahrens. Diese Übelstände drücken die Ausbeute an dem übrigens sehr reinen Ceroxyd sehr herab. Es werden aus dem Oxydgemisch, welches annähernd $45 \%$ Cerdioxyd enthielt, nur 50\% der theoretischen Menge an reinem Oxyd gewonnen.

Hierauf wurde eine weitere Menge des genannten Präparates von Merck nach der Auer von Welsbach'schen Methode, ${ }^{3}$ die, wie erwähnt, von Schotrlafinder genauer studiert worden ist, in Bearbeitung genommen. Diese Methode berubt bekinntlich auf der

\footnotetext{
1 Z. anory. Chenn. 12, 169.

2 Journ. pr. Chem. [2] 12, 209.

- Monatsh. Ohem. 5, 508.
} 
Abscheidung des Cers als Ceriammoniumnitrat. Die Oxyde wurden in starker Salpetersäure gelöst, wobei eine reichliche Sauerstoffentwickelung stattfand. Die Abscheidung als Ceriammoniumnitrat ist sehr einfach und gelingt am besten, wenn man die stark salpetersaure Lösung soweit eindampft, dafs die anfangs dunkelrote Lösung hellgelb wird, während dessen das gewünschte rote Salz in reichlicher Menge sich abscheidet. Diese Methode hat jedoch auch ihre Mängel, nämlich: 1. die schon erwähnte Reduktion beim Lösen des Oxydgemisches in konz. Salpetersäure, wodurch die Ausbeute vermindert wird, und 2. die Unlöslichkeit der Oxyde in Salpetersäure, wenn das Gemisch derselben mehr als ca. $45 \%$ Cerdioxyd enthält. In letzterem Falle müssen die Oxyde mit konz. Schwefelsäure abgeraucht, das Sulfat in Wasser gelöst, mit einer Lösung von Lauthan- oder I)idymsalz versetzt, wodurch der Prozentgehalt an Ceroxyd herabgedrückt wird, und schliefslich von neuem bearbeitet werden, was natürlich umständlich und zeitraubend ist.

Vor längerer Zeit hat Cleve als die beste Methode für die Reindarstellung des Cers die Mosanden'sche ${ }^{1}$ bezeichnet. Nach dieser Methode werden die durch überschüssige Kalilauge gefällten Oxydhydrate mit Chlor behandelt, wobei das Cersesquioxydhydrat in das Dioxydhydrat - seinem Charakter als Polyoxyd entsprechend - übergeführt wird, während die Oxyde des Lanthans, Praseodyms und Neodyms sich in der bei der Reaktion entstehenden Salzsäure lösen. Um nach dieser Methode allein reines Cersalz zu erhalten ist, ein mehrmaliges Wiederholen des Verfahrens erforderlich, da das Cerdioxydhydrat hartnäckig Lanthan und Didym zurückhält. Daher hat O. N. W ITT $^{2}$ die Mosander'sche Methode mit der AoEn'schen kombiniert, indem er das Cerdioxydhydrat von den letzten Spuren des Lanthans und Didyms durch Auflösen desselben in starker Salpetersäure und Abscheidung als Ammoniumdoppelsalz befreit. In der That liefert diese Methode sehr gute Resultate. Sie eignet sich auch sehr gut für den Fall, wenn man cerfreies Didym- und Lanthansalz erhalten will. Schon nach einmaligem Behandeln mit Chlor erhält man ein Gemisch dieser Salze in Lösung, welches keine Spur Cer enthält. Einen Vorzug vor der AUER'schen, für letzteren $Z$ weck angegebenen Methode besitzt dieses Verfahren auch insofern, als schon beim erstmaligen Behandeln

\footnotetext{
1 Journ. pr. Chem. 30, 267.

${ }^{2}$ Chem. Ind. (1896) 19.
} 
fast die ganze Menge der genannten Oxyde erhalten wird, und nur geringe Mengen derselben in den Cerniederschlag mitgehen, was bei dem AuER'schen Verfahren erst nach mehrmaligem Wiederholen der Operation erreichbar ist. Der einzige Übelstand bei dieser Methode ist das lästige Arbeiten mit Chlor, zumal ein befriedigendes Resultat nur nach mindestens 15 stündiger Einwirkung des Chlors erreicht wird.

Als ein sehr geeignetes Oxydationsmittel für die Überführung des Cers in die höhere Oxydationsstufe erwies sica das Natriumsuperoxyd. Man kann hierbei von jedem beliebigen Oxydulsalz des Cers ausgehen. Die Lösung desselben fällt man bei Zimmertemperatur, indem man langsam und unter stetem Umrühren eine Lösung von Natriumsuperoxyd in Eiswasser zugiebt. Der rotbraune, fast eisenoxydfarbene Niederschlag wird mit heifsem Wasser durch Dekantation ausgewaschen und mit der Saugpumpe abgesogen. Das l'rocknen desselben geschieht bei $120-130^{\circ}$, wobei die rotbraune Farbe in Hellbraun bis Gelb übergeht. Der getrocknete Niederschlag wird alsdann in kleinen Portionen in starke Salpetersäure gegeben, worin er sich unter Erwärmung lölst. Hierbei entwickelt sich ziemlich stark Kohlensäure, welche das Oxydhydrat aus der Luft aufgenommen hatte. Eine Reduktion unter Sauerstoffentwickelung, wie beim AUER'schen Verfahren, konnte hierbei nicht nachgewiesen werden. Ess mag dies damit zusammenhängen, dafs das Cerdioxydhydrat sich leichter und unter geringerer Wärmeentwickelung in Salpetersäure löst als das wasserfreie Dioxyd und bei dieser niedrigeren Temperatur seinen Sauerstoff nicht abgiebt. Die Abscheidung des Cers geschieht hierauf als Ammoniumdoppelsalz in bekannter Weise. Der Vorteil dieses Verfahrens vor dem AukR voN WeLsBaCE'schen besteht darin, dals es bei jedem beliebigen Prozentgehalt an Ceroxyd ausfuhrbar ist und vor dem Mosander'schen darin, dafs das Natriumsuperoxyd ein viel bequemeres und rascher zum Ziele führendes Oxydationsmittel ist als das Chlor.

Im folgenden werden die quantitativen Resultate über die Einwirkung von Natriumsuperoxyd auf die Oxyde der Cergruppe mitgeteilt.

\section{Quantitative Versuche.}

Cr.eve ${ }^{1}$ hat durch Fällung mit Ammoniak und Wasserstoffsuperoxyd höhere Oxyde der seltenen Erden von bestimmter $\mathrm{Zu}$ -

'Bl. Soc. Chim. Paris [2] 43, 53. 
sammensetzung erhalten. Seine Resultate konnten bei Anwenilung von Natriumsuperoxyd bei gewöhnlicher Temperatur nicht bestätigt werden. Es wurden zwar Oxydhydrate von höherem Sauerstoffgehalt, als dem Sesquioxyd entspricht, erhalten, die Menge des Sauerstoffs war aber eine bedeutend geringere als die den CuEvE'schen Superoxyden entsprechende und schwankte mit den Versuchsbedingungen, besonders mit der Temperatur. Zu demselben Ergebnis kommt auch KNonRE ${ }^{1}$ bei Anwendung von Ammoniak und Wasserstoffsuperoxyd.

Zunächst wurde reines, nach einem der erwähnten Verfahren dargestelltes Cersalz der Untersuchung unterworfen. Wie schon erwähnt, erhält man beim Fällen mit Natriumsuperoxyd einen braunroten Niederschlag. Die Farbe desselben geht jedoch beim Kochen unter starker Sauerstoffentwickelung allmählich in hellgelb, die Farbe des Cerdioxydhydrats iuber. Die rotbraune Farbe entspricht also ohne Zweifel einem höheren Oxyd des Cers, vielleicht dem Cueve'schen $\mathrm{CeO}_{3}$, welches jedoch bei höherer Temperatur sehr unbeständig sein mufs und bei $100^{\circ}$ vollstäudig in das Dioxyd übergeht. Wie weit die Oxydation mit Natriumsuperoxyd abhängig ist von der Temperatur der Lösung, zeigen folgende Versuche:

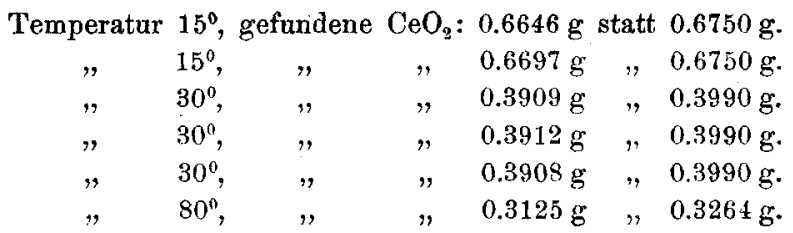

Der Gehalt des Niederschlages an $\mathrm{CeO}_{2}$ wurde mit Ferroammonsalz und Kaliumpermanganat bestimmt. Der Niederschlag darf nicht höher als bei $130^{\circ}$ getrocknet werden, da er schon bei $170^{\circ}$ fast sein ganzes Hydratwasser verliert und dann nicht mehr in Säuren löslich ist. Die angeführten Resultate zeigen, dafs die Oxydation des Sesquioxyds mit Natriumsuperoxyd nicht ganz vollständig ist, was offenbar daher rührt, dafs das entstehende böhere Oxyd Sesquioxyd mechanisch mitreifst und durch Einhüllung vor weiterer Oxydation schützt. Glüht man dagegen den erhaltenen Niederschlag stark auf dem Gebläse, so erbält man die theoretische Menge an Dioxyd, was wiederum beweist, dafs das beste Oxydationsmittel der freie Sauerstoff bei hoher Temperatur ist. Die Resultate, die man

1 Zeitschr. angew. Chem. 1897, 685 und 717. 
auf diese Weise erhält, stimmen genan mit den durch Glühen des Oxalats erhaltenen überein. Das Cerdioxydhydrat löst sich in Salzsäure und Jodkali leicht unter Ausscheidung von Jod, während das wasserfreie Dioxyd dieses nur im zugeschmolzenen Glasrohr beim Erwärmen thut.

Um die Einwirkung von Natriumsuperoxyd auf Lanthan- und Didymsalze quantitativ zu verfolgen, wurde ein cerfreies Gemisch dieser Salze verwandt. Da eine quantitative Trennung, resp. Bestimmung dieser Erden, besonders der Komponenten des Didyms nicht möglich ist, so wurde blofs der Gesamtgehalt der Lösung an Oxyden bestimmt. Die mit Natriumsuperoxyd gefällten Hydroxyde geben beim Erwärmen der Lösung noch stürmischer Sauerstoff ab, als dies beim Cer der Fall war. Bei $120^{\circ}$ getrocknet, hatten sie eine hläulichweifse Farbe und hatten viel Kohlensäure aufgenommen, welche sie erst bei starkem Glühen abgaben. Auch den Sauerstoff halten sie ziemlich stark zurück, wie die folgenden Versuche beweisen:

\begin{tabular}{|c|c|c|c|c|c|c|c|}
\hline $0.6350 \mathrm{~g}$ & Oxyde, & bei & $135^{\circ}$ & etrocknet, & enthielten & noch & $0.0 \mathrm{c}$ \\
\hline $0.6350 \mathrm{~g}$ & ", & " & $230^{\circ}$ & ", & $"$ & $"$ & $0.0034 \mathrm{~g}$ \\
\hline $0.6350 \mathrm{~g}$ & $"$ & " & $500^{\circ}$ & & 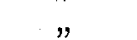 & & 0.0009 \\
\hline $.4305 \mathrm{~g}$ & , & , & $600-700^{\circ}$ & 0 & , & , & 0.0009 \\
\hline
\end{tabular}

Es ist hierbei zu bemerken, dafs bei $400^{\circ}$ ungefähr die bläuliche Farbe der Oxyde in eine dunkelbraune bis braunschwarze übergeht, die erst bei starkem Glühen im Platintiegel auf dem Gebläse in das charakteristische Grau der wasserfreien Sesquioxyde umschlägt. Die grauen Oxyde enthalten nunmehr keine Spur von Kohlensäure und überschülsigem Sauerstoff. Die braune Farbe gehört ohne $Z$ weifel einem höheren Oxyde an, welches von früheren Chemikern als Didymsuperoxyd bezeichnet wurde, nach Aukr voN WeLsBaCH ${ }^{1}$ aber ein höheres Oxyd des Praseodyms $\left(\mathrm{Pr}_{4} \mathrm{O}_{7}\right)$ ist. Das Sesquioxyd des letzteren Elementes hat nämlich die Eigenschaft bei ca. $400^{\circ}$ eine dunkelbraune Farbe unter Bildung von Sauerstoff anzunehmen, welch letzteren es bei starkem Glühen wieder abgiebt. Hierin erweist sich das entstehende höhere Oxyd ähnlich dem Baryumsuperoxyd, und da es aufserdem auch durch Chlor in alkalischer Lösung nicht entsteht, so mufs das Praseodymsuperoxyd

1 Monatsh. Chem. 6, 477. 
zu den Superoxyden des $\mathrm{H}_{2} \mathrm{O}_{2}$-Typus gezählt werden, worauf schon oben hingewiesen wurde.

Die vollständige Analyse einer mit Natriumsuperoxyd gefällten Probe eines Lanthan- und Didymsalzgemisches, welche, nach dem Fällen bei $230^{\circ}$ getrocknet, $0.8318 \mathrm{~g}$ wog, ergab:

$$
\begin{aligned}
& \mathrm{CO}_{2}=0.1365 \mathrm{~g} \\
& \mathrm{H}_{2} \mathrm{O}=0.0500 \mathrm{~g} \\
& \mathrm{O}=0.0034 \mathrm{~g} \text { (nach Analogie einer anderen Probe). }
\end{aligned}
$$

Im ganzen $0.1899 \mathrm{~g}$, d. h. $0.8318-0.1899=0.6419 \mathrm{~g}$ statt $0.6350 \mathrm{~g}$ der geinischten Oxyde.

Beim Glühen der Oxalate von Lanthan und Didym erhält man nicht das braune Oxyd, sondern es entsteht erst weil'ses Karbonat, welches bei starkem Glühen in graues Oxyd übergeht. Befeuchtet man aber letzteres mit Salpetersäure, so erhält man bei vorsichtigem Glühen das sauerstoffreichere braune, bei starkem Glühen wiederum das graue Oxyd. Dasselbe gilt von dem Hydroxyde, das durch Fällen mit Alkalien erhalten wird.

Gemische von Cer, Lanthan und Didym. Die Hoffnung, in Gemischen von den drei Erden den Gehalt an Ceroxyd durch Bestimmung des überschüfsigen Sauerstoffes nach dem Fällen mit Natriumsuperoxyd zu bestimmen, erwies sich als irrig, da auch das Gemisch von Lanthan- und Didymoxyd, bei $230^{\circ}$ getrocknet, noch verhältnismälsig vicl Sauerstoff zurückbält. Durch mehrere Versuche erwies es sich, dal's bei geringem Prozentgehalt an Cer die Resuitate für letzteres zu grols, bei grölserem dagegen zu klein ausfielen. Auch spielt die Temperatur, bei welcher die Fällung ausgeführt wird, dabei eine Rolle, indem in der Kälte gefälltes Oxyd mehr Sauerstoff enthält als heifs gefälltes. Es lag nahe, den überschülsigen Sauerstoff der Lanthan- und Didymoxyde durch starkes Glühen zu entfernen und zugleich etwa noch vorhandenes Cersesquioxyd zu Dioxyd zu oxydieren. Hierbei ergab sich aber die merkwürdige Thatsache, dafs die bei ca. $400^{\circ}$ auftretende und Praseodymsuperoxyd anzeigende Braunfärbung auch bei der höchsten Weifsglut ${ }_{2}$ und lange andauerndem Glühen nicht mehr verschwindet, was bei einem Gemisch von nur Lanthan- und Didymerde doch der Fall war. Übereinstimmend hiermit ergab sich auch der Gchalt an Cerdioxyd, nach dem ïberschülsigen Sanerstoff berechnet, in diesen Fällen als zu grol's, wie folgende Versuche zeigen: 


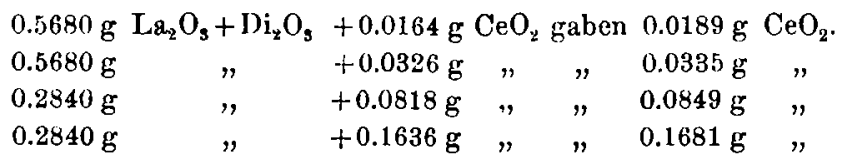

Es ist zu bemerken, dafs im Porzellantiegel auch beim heftigsten Glühen die Werte immer grölser ausfallen als beim Glühen im Platintiegel; im letzteren Falle wird das Gewicht bald konstant. Bei geringem Cergehalte hat das stark geglühte Oxydgemisch eine hellere Farbe, und wird dieselbe dunkler mit steigendem Gehalt an i)idymoxyd. Es folgt hieraus, dafs, wie auch AuER von WeLsBach schon vermutet, das Cerdioxyd mit dem höherem Oxyd des Praseodyms eine in der Hitze beständige Verbindung bildet, in welcher das sauerstoffreichere Superoxyd vielleicht die Rolle einer Säure spielt. Hiermit stimmt auch die in neuester Zeit von Versedul und Wyroubofr' gemachte Annahme überein, dafs das nach allen bisher vorgeschlagenen Methoden dargestellte und für rein gehaltene gelbliche Cerdioxyd noch Spuren einer Verbindung dieses Oxyds mit einer der Komponenten des Didyms enthält.

Beiden Forschern gelang es, vollkommen weilses Cerdioxyd darzustellen. Damit ist auch die Frage nach der Ursache der Braunfärbung eines Gemisches der Oxyde der Ceriterden gelöst. Glüht man nämlich die Oxalate der einzelnen Erden für sich, so erhält man weifse, bezw. graue und gelbliche Oxyde, während ein Gemisch der Oxalate auch in der stärksten Glühhitze ein dunkelbraunes Oxydgemisch liefert, dessen Farbe um so dunkler wird, je mehr man dem Gemisch das Cer entzieht. Die Menge des mit Ferrosalz und Kaliumpermanganat oder mit Salzsäure und Jodkali bestimmbaren Superoxydsauerstoffes ist dabei gröfser als dem Cerdioxyd entspricht, was nur auf Kosten des Praseodymsuperoxyds kommen kann. Folgende Probe wurde mit Oxalsäure gefällt und bis zum konstanten Gewicht auf dem Gebläse im Platintiegel geglüht:

$$
0.2152 \mathrm{~g} \mathrm{La}_{2} \mathrm{O}_{\mathrm{B}}+\mathrm{Di}_{2} \mathrm{O}_{8}+0.1635 \mathrm{~g} \mathrm{CeO}_{2} \text { ergab } 0.1762 \mathrm{~g} \mathrm{CeO}_{2} \text {, }
$$

wobei der Superoxydsauerstoff auf Cerdioxyd umgerechnet wurde und natürlich für letzteres ein zu grofses Resultat ergab. Ferner wurde Merck'sches „Cerium nitric.“, welches $35.54 \%$ Oxyd enthielt, mit Oxalsäure gefällt, geglüht und der Sauerstoff auf Cerdioxyd berechnet, wobei folgende Resultate ${ }^{2}$ erhalten wurden:

1 Compt. rend. 124, 1230.

$\because$ Da die geglühten Oxyde in kalter Lösung von Ferroammonsaly und Schwefelsäure sich nicht lösten, so mulste einige Zeit erwärınt werden. Dals 


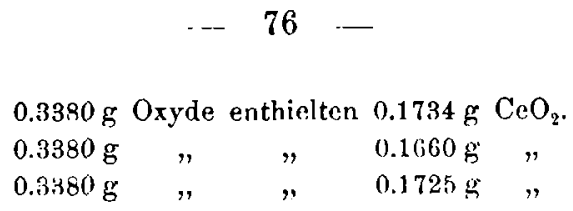

Zwei weitere Proben wurden mit Natriumsuperoxyd heifs gefällt und von diesen die erste bei $175^{\circ}$ getrocknet, die zweite stark geglüht:

1) $0.3380 \mathrm{~g}$ Oxyde exthielten $0.1561 \mathrm{~g} \mathrm{CeO}_{2}$.

2) $0.3380 \mathrm{~g}, \quad, \quad 0.1630 \mathrm{~g}$ "

Bei der ersten Probe wäre das Resultat etwas höher ausgefallen, wenn die Fällung in der Kälte ausgeführt worden wäre. Aus den fünf einigermalsen übereinstimmenden Bestimmungen ergiebt sich, dafs das Oxyd im Mittel kaum 50\% Cerdioxyd enthielt. Man könnte hiergegen einwenden, dafs in dieser Zahl auch das Praseodymsuperoxyd einbegriffen ist. Die Menge des letzteren ist aber sebr gering, wie aus einer oxydimetrischen Bestimmung des Restes der Oxyde, nach vollständiger Entfernung des Cers, hervorging.

Ein Menck'sches „Cerium. oxydat. purum“, von zimmetbrauner Farbe, enthielt im Mittel $42.5 \%$ Cerdioxyd (ans 2 Bestimmungen von 43.03 und 42.05 ).

Jedenfalls sind die oxydimetrischen Methoden für die Bestimmung des Cers in Gemischen der Ceriterden nur dann zulälsig und einigermalsen zuverläfsig, wenn letztere nicht sehr grofse Mengen Praseodymoxyd enthalten, was in der Praxis und bei den Naturprodukten auch meist der Fall ist. Eine genaue Methode für die quantitative Bestimmung des Cers in den Ceriterden besitzen wir somit bis jetzt noch nicht.

Aus der vorhergehenden Kxperimentaluntersuchung ergeben sich folgende Schlüsse:

1. Von allen bis jetzt angewandten Oxydationsmitteln für die Trennung und Reindarstellung des Cers, erweist sich das Natriumsuperoxyd als das bequemste. Diese Methode ist in allen Fïllen anwendbar und liefert die grölste Ausbeute.

2. Durch starkes Glühen der Oxalate der Ceriterden bei Luftzutritt wird nicht nur das Cer vollständig zu Cerdioxyd oxydiert, sondern es entsteht auch das Superoxyd des Praseodyms, welches die braune Färbung des Oxydgemisches bedingt.

das Ferrosalz dabei keine Oxydation an und für sich erleidet, wurde durch Parallelversuche sichergestellt. 
3. Während ein Gemisch von Lanthan- und Didymoxyd, welches bei $400-500^{\circ}$ unter Braunfürbung Sauerstoff aufnimmt, letateren bei Weifsgluth wieder abgiebt und dabei eine graue Farbe annimmt, tritt letzteres nicht ein, wenn Cer zugegen ist, was auf eine in der Hitze beständige Verbindung zwischen Ceroxyd und Praseodymsuperoxyd hinweist.

4. Auf Grund der beiden vorhergehenden Thatsachen sind die oxydimetrischen Methoden für die quantitative Bestimmung des Cers in den Ceriterden, wenn die Oxyde an der Luft geglüht wurden, nicht anwendbar und liefern nur dann annähernde Werte, wenn nicht viel Praseodym zugegen ist, welche Bedingung in der Praxis meist erfüllt ist.

Riga, Polytechn. Institut, im September 1898.

Bei der Redaktion eingegangen am 4. Oktober 1898. 